\title{
NURBS-BASED ISOGEOMETRIC ANALYSIS FOR BALLISTIC EVALUATION OF TITANIUM PLATES
}

\author{
Mattia Montanari $^{1}$, Petros Siegkas ${ }^{1}$, Antonio Pellegrino $^{1}$, Nik Petrinic ${ }^{1}$ \\ ${ }^{1}$ Department of Engineering Science, University of Oxford \\ Parks Road, OX1 3PJ, Oxford, UK \\ e-mail: mattia.montanari@eng.ox.ac.uk
}

Keywords: Isogeometric analysis, NURBS, Impact testing, Titanium alloy, High strain rate.

\begin{abstract}
The variation diminishing property of non-uniform rational B-splines (NURBS) makes NURBS-based isogeometric analysis attractive for applications involving travelling stress waves generated, for example, by impulsive loads. Over their finite elements counterparts, the advantage of NURBS basis functions is the higher continuity at every point of single-patch domains. This research investigates the applicability of isogeometric analysis for the ballistic performance of titanium alloys. Numerical results, in the framework of explicit time-integration, present the effect for $k$-refined trivariate NURBS. A comparison with the classical finite element analysis is also presented. Although the global continuity of NURBS results into a superior approximation of the travelling waves, it does not allow physical discontinuities in the displacement field. This prevents, for example, a projectile to penetrate a target plate. It is argued that the realistic physical response can be captured by adopting material models incorporating strain-rate-sensitive failure criteria.
\end{abstract}




\section{INTRODUCTION}

Ballistic tests provide greater insight into the dynamic response of mechanical components. Usually, ballistic tests aim to measure the ballistic limit of a target: the threshold velocity for which penetration occurs. Nowadays, these tests are not restricted to military applications; bird-strike and safety equipment tests are only few of the many case studies involving ballistic experiments.

The complexity and the high costs of experimental test are often a limitation to the accurate measurement of the ballistic limit. Numerical simulations provide tangible benefits and are, in fact, an integral part of the design process for mechanical components undergoing ballistic loads. The general tool adopted in many engineering communities is the finite element analysis (FEA). Several works using this method have been published in the fields of aerospace and mechanical engineering to model ballistic tests on steel [1], woven fabric [2] and titanium plates [3]. We now want to expand these studies to the a higher-order approximation method by means of isogeometric analysis.

Isogeometric analysis (IGA) [4] is a numerical paradigm that has several advantages compared to the classical FEA. Firstly, by employing non-uniform rational B-splines (NURBS) as basis functions, IGA avoids the geometrical inaccuracies introduced by the FEA meshes. Moreover, spline provide a superior approximation to stress travelling waves, making IGA appealing for dynamic simulations.

During perforation, FEA models are usually coupled with element erosion techniques that have major drawbacks [5, 6]. By taking away mass from the system, these methods inevitably compromise the inertia and thus the dynamic response of the system itself. If the features of spalled plug or residual crater are not of primary interest, and if the ultimate goal of the investigation is to predict whether penetration would occur or not, element erosion can be avoided altogether. More accurate numerical methods allowing for cracks growth have been proposed in the literature. These approaches face enormous computational challenges as they have to take into account for geometrical instabilities and discontinuities [7]. The extended finite element method (X-FEM) [8], reproducing kernel particle method (RKPM) [9] and phase field models [10] are just few of the well-established approaches available in the literature. Nevertheless, the advantages of such methods come at the price of a higher computational cost. For this study a pragmatic and closer to in-service engineering applications is pursued.

To predict the ballistic limit we propose to combine IGA with a material model with failure criterion. For this work the constitutive model developed by Børvik et al [11] was selected. Originally formulated starting from the Johnson-Cook [12] material model and the continuum damage mechanics model by Lamaitre [13], this is one of the most diffused constitutive models for the simulation of ballistic tests. At last, we provide a comparison of numerical results obtained with IGA and FEA.

\section{NURBS-BASED ISOGEOMETRIC ANALYSIS}

Higher-order NURBS shape function have a great advantage over their finite element counterparts, that is, splines do not oscillate nor overshoot near discontinuities. Instead, higherorder polynomials yield to the so-called Gibbs phenomenon [14]. Another advantage is that NURBS are found in the every computer aided design (CAD) software packages for engineers. NURBS-based IGA represents CAD models exactly and thus reduces the gap between CAD and numerical analysis. 
A NURBS curve of order $p$ is defined over a one-dimensional parametric domain as:

$$
\mathbf{C}(u)=\frac{\sum_{i=0}^{n} N_{i, p}(u) w_{i} \mathbf{P}_{i}}{\sum_{i=0}^{n} N_{i, p}(u) w_{i}} \quad 0 \leq u \leq 1
$$

The control points $\mathbf{P}_{i}$ map the curve from parametric to Euclidean three-dimensional space $\mathbb{R}^{3}$. To each control point is associated a weight $w_{i}$ which, together with the B-spline basis function $N_{i, p}(u)$, defines the rational basis function $R_{i, p}(u)$ such that:

$$
\mathbf{C}(u)=\sum_{i=0}^{n} R_{i, p}(u) \mathbf{P}_{i}
$$

The most common formulation of B-spline basis function for computer application is the recursive Cox-de Boor [15] formula:

$$
\begin{aligned}
& N_{i, 0}(u)=\left\{\begin{array}{rr}
1 & \text { if } \quad u_{i} \leq u<u_{i+1} \\
0 & \text { otherwise }
\end{array}\right. \\
& N_{i, p}(u)=\frac{u-u_{i}}{u_{i+p}-u_{i}} N_{i, p-1}(u)+\frac{u_{i+p+1}-u}{u_{i+p+1}-u_{i+1}} N_{i+1, p-1}(u)
\end{aligned}
$$

The functions in Eqs. (1) and (2) are defined on a knot vector $\Xi$, which is a nondecreasing and nonuniform sequence of real numbers between 0 and 1. Each entry is a knot, and each knot has multiplicity at the most $p+1$. Only the extremes of $\Xi$ have multiplicity necessarily equal to $p+1$. For example, a general knot vector may be written as

$$
\Xi=\{\underbrace{0, \ldots, 0}_{\mathrm{p}+1}, \xi_{1}, \ldots, \xi_{m}, \underbrace{1, \ldots, 1}_{\mathrm{p}+1}\}
$$

For the tests presented in this work, the set of knots $\left\{\xi_{j}\right\}$ is obtained by evaluating the equation below at $m=n-(p+1)$ equally distributed points between $-a$ and $a$.

$$
\xi_{j}=\frac{\tan t_{j}}{2 a}+0.5 \quad t \in[-a, a] \quad j=1, \ldots, m
$$

For the present study $a=7 \pi / 3$. Figure 1 shows an example of NURBS curve with knot vector obtained from Eq. (5) for $m=3$ and $w_{i}=1$ for all $i=1, \ldots, n$.

The curve $\mathbf{C}(u)$ inherits a series of properties from its basis functions. The functions $R_{i, p}(u)$ form a partition of unity $\left(\sum_{i=0}^{n} R_{i, p}(u)=1 \forall u\right)$ and have compact support $\left(R_{i, p}=0\right.$ if $\left.u \notin\left[u_{i}, u_{i+p+1}\right)\right)$. Similar properties exist for the Lagrange polynomials used in FEA. The difference is that the rational function $R_{i, p}(u)$ are nonnegative $\left(R_{i, p}(u) \geq 0 \forall i, n\right.$ and $\left.0 \leq u \leq 1\right)$, do not necessarily interpolate the Kronecker delta functions [14] and benefit from the variation diminishing property [16].

The most striking consequence of the latter property is the absence of Gibbs phenomenon for higher-order spline interpolants. This is a spurious numerical oscillation that occurs when a sharp discontinuity (e.g. the step function) is approximated by a high-order polynomial. The inefficiency of Lagrange polynomials to describe jumps and discontinuities is inevitably seen in the classic FEA. The variation diminishing property of NURBS curves establishes that a plane cannot intersect more times the curve $\mathbf{C}(u)$ than control polygon. Intuitively, this property ensures that a NURBS curve does not oscillate more than its control grid. 


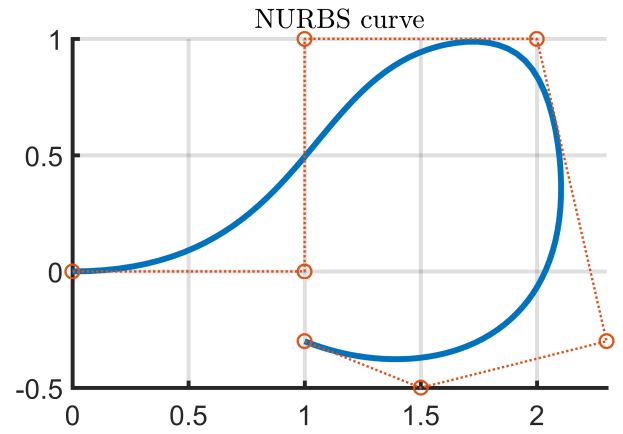

(a)

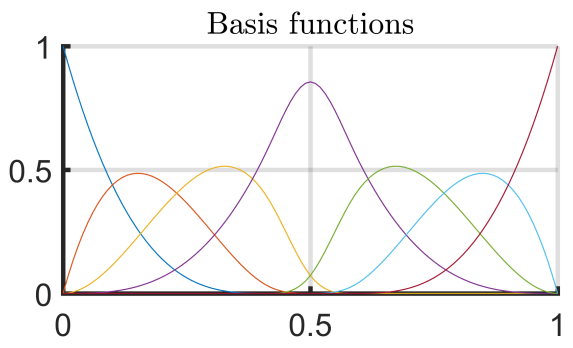

(b)

Figure 1: (a) Cubic NURBS curve $\mathbf{C}(u)$ defined over the knot vector $\Xi=[0,0,0,0,0.3819,0.5,0.6181,1,1,1,1]$ illustrated with its control grid. (b) Representation of the basis functions $N_{i, p}(u)$ of $\mathbf{C}(u)$.

From the tensor product of three NURBS curves, a trivariate solid element may be obtained. This is a vector-evaluated function of three parameters. Each parametric variable may have an order $p$ and an $h$-refinement different from the other two. As a result, tensor product NURBS solids can be illustrated as a solid cube in parametric domain. By mapping the parametric cube one can, for example, obtain the uniaxial test specimen in Figure 2. For this study the parametric cube was mapped into a simple plate.

It should be noted that the variation diminishing property does not exists for NURBS surfaces and solids. For these, however, the property applies somewhat in a weaker sense. Reference is made to [18] for NURBS and to [19] for NURBS-based IGA.

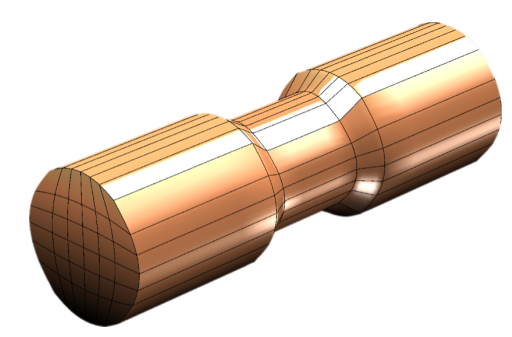

Figure 2: Uniaxial test specimen model described by tensor product solid NURBS.

\section{NUMERICAL MODEL}

\subsection{Description}

The numerical model of the ballistic test includes a target, a rounded nose projectile and four semi-spherical supports. Figure 3 illustrates the model at time $t=0$. For this model the effect of friction is considered negligible and a rigid body assumption is made for the projectile and the supports. These assumptions will have a minor effect on the predicted physical response, but do not cancel the outcome of the comparison between IGA and FEA.

The projectile impacts the target at a velocity of $400 \mathrm{~m} / \mathrm{s}$, which is well above the ballistic limit of the plate. The target is a $7 \mathrm{~mm}$ thick, $127 \mathrm{~mm}$ wide titanium plate mounted onto four fixed supports. The mass of the projectile is equal to $67.3 \mathrm{~g}$. The contact between the plate, supports and projectile uses a standard penalty master-slave formulation. 


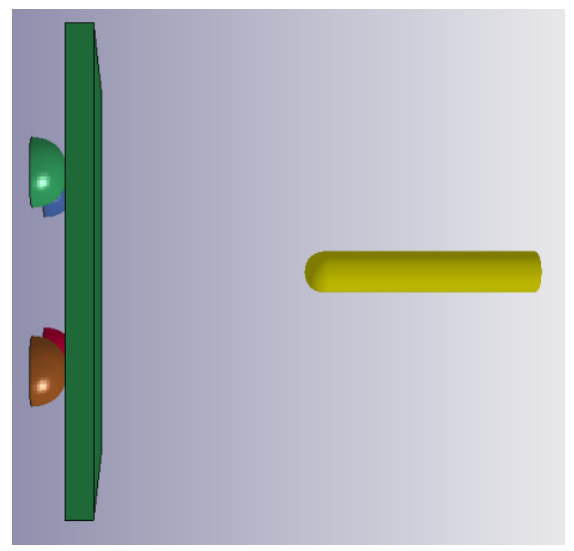

Figure 3: Perspective view of the ballistic test numerical model

\subsection{Spatial discretization}

For the discretization of the titanium plate, IGA uses a single solid NURBS patch, while FEA employs solid brick elements. The starting point to produce the NURBS patch is a quadratic trivariate model (Figure 4a). If a higher-order approximation is required, the order of this reference patch is elevated accordingly. Afterwards, the NURBS is $h$-refined by knot insertion. The knots inserted in the two parametric direction creating the face of the plate are defined by Eq. (5). The three models depicted in Figures 4b $4 \mathrm{~d}$ (only the top-right quarter of each model is shown), are obtained for values of $m=10,15,23$ respectively.

The FEA model employs Lagrange first-order polynomials to describe the titanium plate. For the numerical solution under-integrated hexahedral finite element are used. After a mesh sensitivity study, it was observed that a mesh with approximately 20000 elements would have generated convergent results in terms of projectile velocity after perforation. However, a finer mesh with about the double of number of elements was used to mitigate the hourglass effect. Detailed information about the spatial discretizations are presented in Table 1.

\subsection{Material model}

In this work we use the material model proposed by Børvik et at [11]. This constitutive model couples viscoplasticity with ductile damage and was designed for modelling impacts that exhibit penetration. Some application of this model that can be found in literature are [17, 20].

The model describes the equivalent stress using the following equation

$$
\sigma=\left(A+B \bar{\varepsilon}_{p}^{n}\right)\left(1-\frac{T-T_{r}}{T_{m}-T_{r}}\right)\left(1+\frac{\dot{\bar{\varepsilon}}_{p}}{\dot{\varepsilon}_{0}}\right)^{C}
$$

where $A, B, C, n, m$ are material constants. The accumulated plastic strain is $\bar{\varepsilon}_{p}$, the equivalent strain rate and the reference strain rate are $\dot{\bar{\varepsilon}}_{p}, \dot{\varepsilon}_{0}$, respectively. The second term in the equation above considers the temperature effect; $T$ is the absolute temperature, while $T_{r}$ and $T_{m}$ are room and melting temperatures, respectively.

This model couples damage with the constitutive equation by means of following the fracture strain law:

$$
\varepsilon_{f}=\left[D_{1}+D_{2} \exp \left(\frac{D_{3} \sigma_{m}}{\bar{\sigma}}\right)\right]\left[1+\frac{\dot{\bar{\varepsilon}}_{p}}{\dot{\varepsilon}_{0}}\right]^{D_{4}}\left[1+D_{5} \frac{T-T_{r}}{T_{m}-T_{r}}\right]
$$




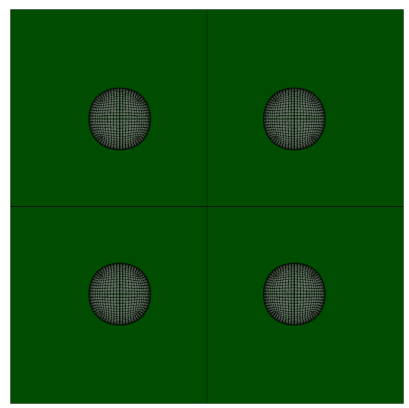

(a)

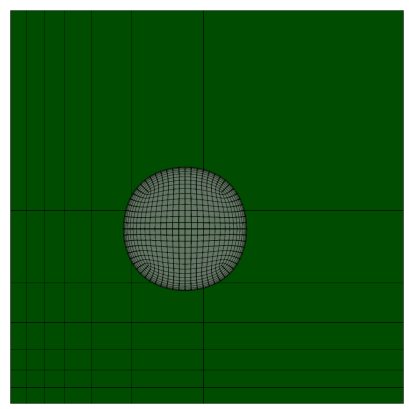

(c)

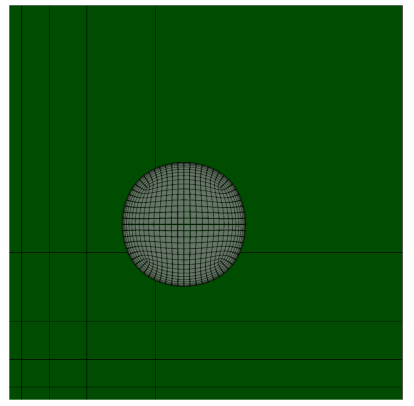

(b)

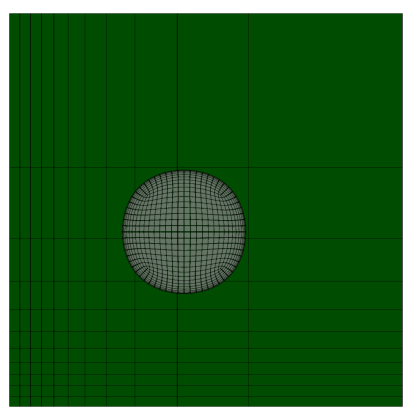

(d)

Figure 4: Spatial discretization for the IGA model. (a) Backside view with supports and NURBS patch. (b)-(d) Top-right quarters of the model after various $h$-refinements.

This is an empirical formulation for which the material constants $D_{1}, \ldots, D_{5}$ need to be calibrated experimentally. The evolution damage rule is relatively simple and given as

$$
\dot{D}=\left\{\begin{array}{ccc}
0 & \text { for } \quad \bar{\varepsilon}_{p} \leq \varepsilon_{d} \\
D_{c} \frac{\dot{\bar{\varepsilon}}_{p}}{\varepsilon_{f}-\varepsilon_{d}} & \text { for } & \bar{\varepsilon}_{p}>\varepsilon_{d}
\end{array}\right.
$$

where $\varepsilon_{d}$ is the value of the damage threshold. The material fails as the damage parameter $D$ reaches the critical value $D_{c}$.

The advantages of this constitutive model are the simple calibration and its computational efficiency. An experimental campaign was conducted in our laboratory to obtain the material parameters which will be disclosed in a future publication.

\subsection{Results}

Numerical tests are carried out using the commercial software LS-DYNA. This allows to use solid NURBS element as well as the material model described in the previous section. The velocity of the projectile and the stress state of the target are the main outcomes of the numerical tests presented in this work.

Herein, we present the numerical results for an initial velocity of the projectile $v=400 \mathrm{~m} / \mathrm{s}$. All tests, for both IGA and FEA, predicted penetration. The results are summarised in Table 1 .

Figure 5 shows the velocity of the projectile for both IGA and FEA. The comparison do not show good agreement. FEA simulation result in a much lower velocity after penetration. In this sense IGA produces somewhat conservative results. At first sight this might be attribute to volumetric locking effect. It is however interesting to observe that NURBS basis functions of order $p=3$ (test 052 ) approximate the FEA solution better than those of $p=2$ order. 


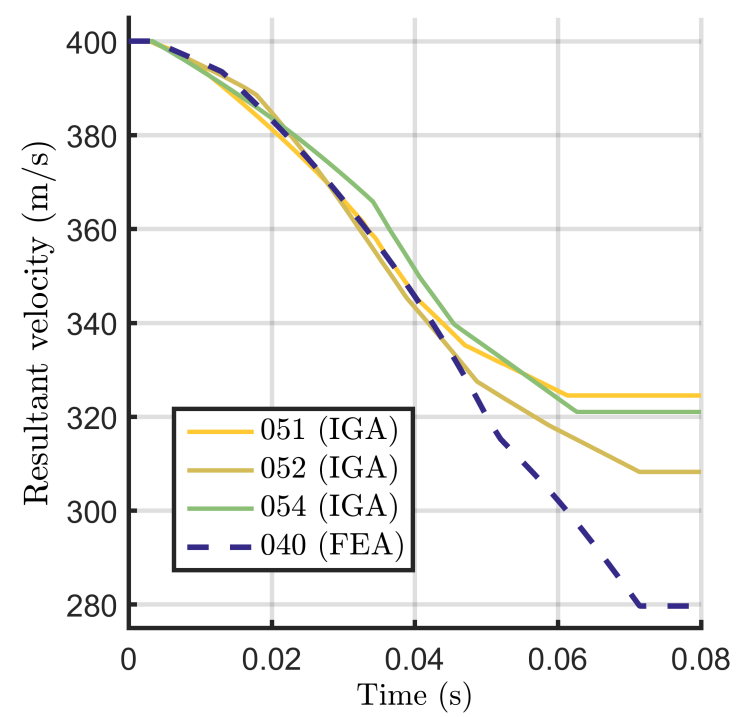

Figure 5: Comparison between second-order IGA (tests 051 and 054), third-order IGA (test 052) and FEA (test 040).

Table 1: Results for projectile initial velocity $v=400 \mathrm{~m} / \mathrm{s}$.

\begin{tabular}{ccccccc}
\hline Test ID & Method & $p$ & $m$ & Nodes & $v_{\text {final }}(\mathrm{m} / \mathrm{s})$ & $\mathrm{CPU}_{\text {time }}(\mathrm{h})$ \\
\hline 051 & IGA & 2 & 10 & 726 & 324.5 & 23 \\
052 & IGA & 3 & 10 & 1008 & 308.2 & 40 \\
054 & IGA & 2 & 15 & 2048 & 321.0 & 61 \\
040 & FEA & 1 & - & 40368 & 279.7 & 64 \\
\hline
\end{tabular}

The stress state of the target plate for the IGA test 052 is illustrated in Figure 6. The backside view of four solution frames represent: supports, deformed meshes (with magnified displacement) and von Mises stress contour plots. It can be seen that, as the simulation evolves, the stress builds up (Figures $6 \mathrm{a}$ and $6 \mathrm{~b}$ ). Once failure emerges, the stress in the damaged area drops (Figure 6c) until complete penetration of the plate (Figure 6d). 


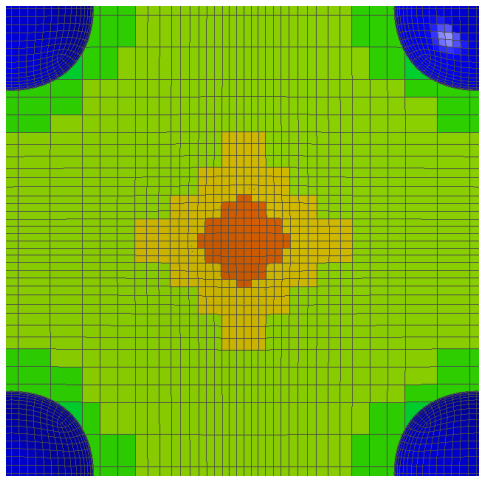

(a)

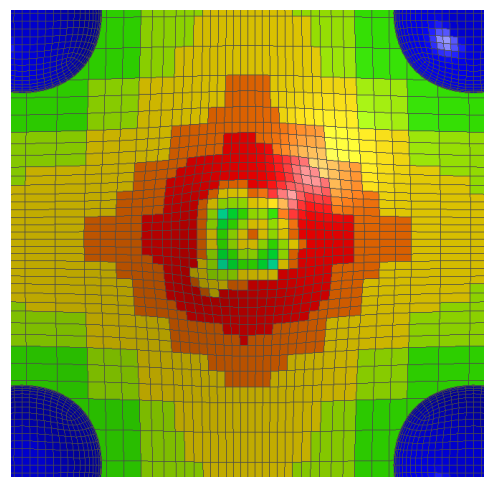

(c)

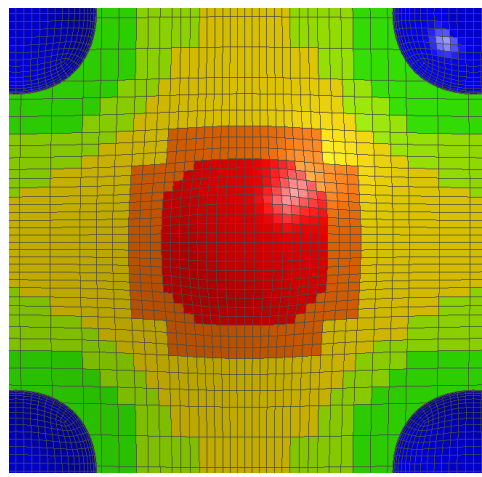

(b)

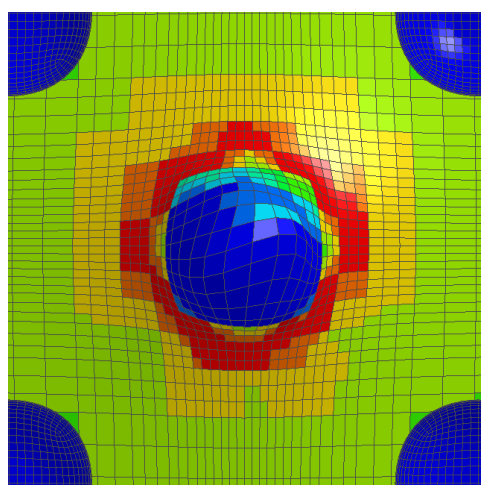

(d)

Figure 6: Von Mises stress contour plots from the backside view of the target at different time frames: (a) $t=0.0009$, (b) $t=0.0386$, (c) $t=0.0460$ and (d) $t=0.0800$. The red colour corresponds to stress of $800 \mathrm{MPa}$ and above, while the blue colour indicates zero stress. 


\section{CONCLUSIONS}

This study presents preliminary results of a ballistic test model that couples IGA with continuum damage mechanics. The numerical results are compared with classic FEA. For both methods mesh sensitivity is preformed and the constitutive model successfully predicts failure of the titanium target plate.

When penetration occurs the dynamic responses predicted by IGA and FEA diverge. Given the different nature and properties of the basis functions employed by the two methods, this difference is justifiable. An experimental validation, together with a systematic study on volume locking effects, will be addressed in future works.

\section{ACKNOWLEDGEMENTS}

The authors gratefully acknowledge the financial support of this research by the UK Technology Strategy Board and Rolls-Royce plc through the Strategic Investment in LOw-carbon Engine Technology (SILOET 2) project.

\section{REFERENCES}

[1] Iqbal, M.A. , Senthil, K. , Bhargava, P. ,Gupta, N.K. (2015). The characterization and ballistic evaluation of mild steel, International Journal of Impact Engineering, 78:98-113.

[2] Ercan Sevkat, (2012). Experimental and numerical approaches for estimating ballistic limit velocities of woven composite beams, International Journal of Impact Engineering, 45:16-27.

[3] Hubert W. Meyer Jr., Kleponis, D. S. (2001). Modeling the high strain rate behavior of titanium undergoing ballistic impact and penetration, International Journal of Impact Engineering, Volume 26:509-521.

[4] Hughes, T. J. R. , Cottrell, J. A. , and Bazilevs, Y. (2005). Isogeometric analysis: CAD, finite elements, NURBS, exact geometry and mesh refinement Computer Methods in Applied Mechanics and Engineering, 194:4135-4195.

[5] Chen E.P. (1992). Numerical simulation of shear induced plugging in HY100 steel plates. International Journal of Damage Mechanics, 1:132-143.

[6] Sewell, D. A., Ong, A. C. J. , Hallquist, J. (1990). Penetration calculations using an erosion algorithm in Dyna. Proceedings of 12th International Symposium on Ballistics, San Antonio, USA.

[7] Belytschko, T., Liu, W. K., Moran, B. , Elkhodary, K., (2013). Nonlinear Finite Elements for Continua and StructuresJohn, Wiley \& Sons.

[8] Mos, N., Dolbow, J., Belytschko, T. (1999). A finite element method for crack growth without remeshing. International Journal for Numerical Methods in Engineering, 46:131150 .

[9] Barbieri, E., Petrinic, N., (2013). Three-dimensional crack propagation with distancebased discontinuous kernels in meshfree methods Computational Mechanics, 53:325-342. 
[10] Borden, M. J., Hughes,T. J. R., Landis, C. M., Verhoosel, C. V., (2014). A higher-order phase-field model for brittle fracture: Formulation and analysis within the isogeometric analysis framework, Computer Methods in Applied Mechanics and Engineering, 273:100118.

[11] Børvik T., Hopperstad, O. S., Berstad, T., Langseth, M., (2001). A computational model of viscoplasticity and ductile damage for impact and penetration, European Journal of Mechanics - A/Solids, 20:685-712.

[12] Johnson, G.R., Cook, W.H., (1983). A constitutive model and data for metals subjected to large strains, high strain rates and high temperatures.Proceedings of Seventh International Symposium on Ballistics.

[13] Lemaitre, J., Chaboche, J. L. (1990). Mechanics of Solid Materials. Cambridge University Press.

[14] Trefethen, Lloyd N. (2013). Approximation theory and approximation practice, SIAM.

[15] De Boor, C. (1978). A Practical Guide to Splines. Springer-Verlag.

[16] Schoenberg, I.J., Greville, T.N.E., (1967). On spline functions. Inequalities, 1:255-291.

[17] X.G. Li, X.-L. Gao, S. Kleiven, (2016). Behind helmet blunt trauma induced by ballistic impact: A computational model, International Journal of Impact Engineering, 91:56-67

[18] Piegl L., Tiller, W., (1997). The NURBS Book. Springer.

[19] Cottrell, J. A., Hughes, T. J. R. , and Bazilevs, Y., (2009). Isogeometric Analysis: Toward Integration of CAD and FEA. John Wiley \& Sons.

[20] Børvik, T.,Hopperstad, O. S., Dey, S., Pizzinato, E. V., Langseth, M., Albertini, C., (2005) Strength and ductility of Weldox $460 \mathrm{E}$ steel at high strain rates, elevated temperatures and various stress triaxialities. Engineering Fracture Mechanics, 72:1071-1087. 